\title{
Reduction over Coset Spaces and Residual Gauge Symmetry
}

\section{Davis $\mathbf{S}^{*}$}

Research Foundation of Southern California, 8837 Villa La Jolla Drive \#13595 La Jolla, California, USA

\begin{abstract}
The reduction of higher-dimensional theories over a coset space $S / R$ is known to yield a residual gauge symmetry related to the number of $R$-singlets in the decomposition of $S$ with respect to $R$. It is verified that this invariance is identical to that found by requiring that there is a subgroup of the isometry group with an action on the connection form that yields a transformation rule defined only on the base space. The Lagrangian formulation of the projection of the frame of global vector fields from $S^{7}$ to the Lie group submanifold $S^{3} \times S^{3}$ is considered. The structure of an octonionic Chern-Simons gauge theory is described.
\end{abstract}

Keywords: Fibre bundle; Dimensional reduction; Centralizer; Super symmetry

\section{Introduction}

The geometrical formulation of classical Yang-Mills theory through principal bundles requires a fibre that is diffeomorphic to the structure group. Matter fields may be described by an associated vector bundle with the vector space forming a representation of the structure group. The geometry of a quantum theory and its anomalies has been developed by considering jet bundles with tangent vectors and higherorder derivatives to the fibre.

The geometrical description of a Lagrangian field theory through fibre bundles has been achieved for Yang-Mills theories with arbitrary Lie groups representing gauge symmetries. The gauge field action may be expressed as the integral of the curvature form where the gauge potential is the connection of the bundle, while the actions of other matter fields consist of integrals of inner products of sections of vector bundles, where the vector space forms a representation of the gauge group [1]. This formalism has been extended to discrete groups [2].

Classical field theories may be formulated through tangent bundles with the Lagrangian invariant under transformations generated by vector fields along the fibre [3]. The characteristic classes of the principal bundle yields a sequence of topological gauge actions, which include the integral of $\operatorname{Tr}(F \wedge F)$ on a four-dimensional manifold that equals the Chern-Simons action on the boundary [4]. Generalized Chern-Simons forms have been given in a recursion formula in terms of the connection form [5].

The types of fibres which admit a connection transformation rule for a theory defined only on the base space must admit a global parallelism and have structure functions that are constant, requiring a quotient of a Lie group by a discrete subgroup [6]. When the fibre of a bundle is not a Lie group, the action of the isometry group of the fibre on the connection form yields a transformation rule that generally includes a fibre coordinate dependence. This generalized transformation reduced to the conventional rule for a subgroup such that the potentials are defined entirely on the base space.

Two methods for generalizing the transformation of the connection form in a principal bundle have been developed. The first, considered in this investigation, requires the existence of a transformation of the connection form, with values in the vertical subspace of the tangent space to the total space, under the isometry group of the fibre. The second consists of a projection of the vector fields on the fibre to a group submanifold. The example of the projection of the parallelism on
$S^{7}$ to $S^{3} \times S^{3}$ provides a theoretical basis [6-8] for the effective number of vector bosons in the theory of the strong interactions [9]. The latter technique does not begin with an action, which may follow from a consideration of the initial formalism.

The dimensional reduction of a theory over a coset $S / R$ can be achieved if the fields are invariant under $\mathrm{S}$. The decomposition of the vector space representation of $S$ into representations of $R$ consists of singlet and nonsinglet states. The residual symmetry is determined by the number of singlet representations [10]. Equivalence with the subgroup derived by the action of the isometry group on the fibre would follow if the nonsinglet states have indices that must represent coordinates in the direction of the coset, and it is proven in $\S 2$. Therefore, a relation exists between the phenomenological model of the elementary particle forces with a geometrical description through fibre bundles and a Lagrangian field theory.

Generally, actions with an $S^{7}$ symmetry do not exist in closed form because the antisymmetric combination of the structure constants that vanishes for a Lie algebra would equal a four-index symbol representing the nonassociativity of the octonions. There are other identities, however, which relate the products of structure constants and the four-index symbols or the products of the four-index symbols with the original set of constants. An integral equivalent to the ChernSimons action is demonstrated to be vary under transformations with generators proportional to the Gell-Mann matrices that can be identified the elements of the octonion algebra into an integral with this antisymmetric combination of structure constants. In the fibre bundle formulation, there is another integral of an inner product consisting of three vector fields on $S^{7}$ transformations, which would transform in an integral consisting of global vector fields under a transformation represented by the Lie derivative along any global $S^{7}$ vector field. Nevertheless, the four-index symbol will appear in the combination of vector fields in the variation of the interaction term and cause the action not to be invariant. The resolution of the absence of closure

${ }^{*}$ Corresponding author: Davis S, Research Foundation of Southern California, 8837 Villa La Jolla Drive \#13595 La Jolla, California-92039, USA, Tel: 91362-7354; E-mail: sbdavis@resfdnsca.org

Received May 30, 2016; Accepted July 09, 2016; Published December 30, 2016

Citation: Davis S (2016) Reduction over Coset Spaces and Residual Gauge Symmetry. J Generalized Lie Theory Appl 10: 249. doi:10.4172/1736-4337.1000249

Copyright: (c) 2016 Davis S. This is an open-access article distributed under the terms of the Creative Commons Attribution License, which permits unrestricted use, distribution, and reproduction in any medium, provided the original author and source are credited. 
under the variational transformations is given in $\S 3$ by a generalized Chern-Simons action consisting of an infinite number of interaction terms constructed with an $S^{7}$ symmetry. This symmetry differs from $S^{7}$ symmetries occurring within larger algebras such as the $\hat{S}^{7}$ algebra of invariances of the heterotic string theory [11-13]. The residual symmetry groups in a bundle with a fibre diffeomorphic to $S^{7}$ would represent gauge symmetries that result from a reduction sequence in a unified field theory with the bosons transforming under an $S^{7}$ symmetry and the fermions belonging to the fundamental representation of the isometry group of an $(S U(3) \times U(1)) /\left(U(1)^{\prime} \times U(1)^{\prime \prime}\right)$ manifold [3] under the gauge theoretical correspondence between $G$-invariant connections on $G / H$ and principal bundle connections on $N(H) / C(H)$ [14], where $G=S U(4)$ and $H=S U(3)$ and $U(1)^{\prime} \times U(1)^{\prime \prime}$ represents the different embeddings of $U(1) \times U(1)$ in $S U(3) \times U(1)$.

\section{The Residual Symmetry Group}

The relation between generalized dimensional reduction and the factorization of the fibre coordinate from the connection form transformation rule will be proven for fibre bundles with fibres that are coset space $S / R$, where $S$ is the isometry group and $R$ is the stabilizer. A vector field on $S / R$ is $S$-invariant if $V^{\mu}(s(x))=J^{\mu}(s, x) V^{\rho}(x)$, where $J^{\mu}$ form a representation of $S$. By regarding an element of $S$ as $\exp (\tilde{z}) r$ where $r \in R$ and $\tilde{z} \in \tilde{Z}$, the Lie algebra in the sum $\tilde{S}=\tilde{R}+\tilde{Z}$. and mapping the fields to a neighbourhood of the identity, it follows that R-invariance at the base point is necessary and sufficient for S-invariance over the coset space. Letting $j^{\mu}(r)=J_{\rho}^{\mu}(r, 0)$, the R-invariance in this neighbourhood is given by $\left.V^{\mu}(y)\right)^{\rho} j_{\rho}^{\mu}(r)^{\rho} V^{\rho}(y)[10]$.

Since nontrivial irreducible representations of $\mathrm{R}$ will leave invariant the vectors on $S / R$, the components of the vector fields on the base space are those that transform as singlets under R. A decomposition of the adjoint representation of $S$ into irreducible representations of $R$ will provide the gauge fields in the base space after dimensional reduction.

The examples of the seven-sphere as the coset spaces $\mathrm{SO}(8) / \mathrm{SO}(7)$ and $\mathrm{SU}(4) / \mathrm{SU}(3)$ will be considered.

\section{Proposition 2.1}

There are no trivial representations in the decomposition of $\mathrm{SO}(8)$ with respect to $\mathrm{SO}(7)$ representations. The number of conditions resulting from the independence of the connection transformation rule for $\mathrm{SO}(8) / \mathrm{SO}(7)$ with respect to the fibre coordinate is finite. It exceeds the number of elements of the gauge matrix in the homogeneous term and the the residual gauge symmetry is given by the identity element.

Proof: The decomposition of the adjoint representation of the isometry group of the round seven-sphere is $28 \rightarrow 21 \oplus 7$. The absence of a singlet $\mathrm{SO}(7)$ representation in the decomposition of the adjoint representation of $\mathrm{SO}(8)$ causes there to be no residual gauge invariance in the four-dimensional theory after reduction from eleven dimensions [10].

The identical invariances are found when the transformation rule of the connection form is generalized to that of the action of an isometry group. If $\sigma(x)$ represents the section of bundle $E(M, G, F, \pi)$ and $\sigma^{\prime}(x)=\sigma(x) \cdot g(x)$, then

$$
\sigma_{*}^{\prime} \cdot \xi=R_{g^{*}} \sigma_{*} \cdot \xi+L_{(y \cdot g)^{*}} L_{g^{-11_{*}}}\left(g_{*} \cdot \xi\right)
$$

and

$$
\omega\left(\sigma_{*}^{\prime} \cdot \xi\right)=L_{(y \cdot g)^{*}}^{-1} R_{g^{*}} L_{y^{*}} \omega\left(\sigma_{*} \cdot \xi\right)+L_{g^{-1 *} g_{*}} g_{*} \cdot \xi .
$$

With $g_{*} \xi$ being the lift of the vector $\xi_{\mu}$ to the tangent space to the fibre at the coset $g(x) H[7]$.

The independence of the in homogeneous term with respect to the fibre coordinate derived from the action of $\mathrm{SO}(8)$ on $S^{7}$ would follow from that of the row vector $y \cdot\left(d_{A B} J_{A B}\right) \cdot\left(t_{L}\left(y^{-1}\right)^{T}\right)$, where $t_{L}: S^{7} \rightarrow S O(8)$ is defined by $y y^{\prime}=y^{\prime} t_{L}(y)$ and $\left\{J_{A B}\right\}$ is the set of generators of $\mathrm{SO}(8)$ given by

$$
\begin{array}{r}
\left(J_{A B}\right)_{i j}=\left\{\begin{array}{l}
1 \\
-1
\end{array} \quad i=A, j=B, j=A\right. \\
0 \quad i, j \neq A, B
\end{array}
$$

Identifying $y$ with $g(x) H, \lambda_{y^{*}}$ is a composite mapping

$$
\begin{aligned}
& T_{e}(G) \rightarrow T_{g(x)}(G) \\
& \downarrow \\
& T_{g(x) H}(F)
\end{aligned}
$$

And $t_{L}\left(y^{-1}\right)^{T}: T_{g(x) H}(F) \rightarrow T_{e}(F)$. At the identity $e=(1,0,0, \ldots, 0)$, the tangent vector is $\left(0 c_{2} c_{3} c_{4} c_{5} c_{6} c_{7}\right)$. The twenty-one constraints on the coefficients $d_{A B}$ require the remaining generators to be

$$
\begin{aligned}
& X_{1}=J_{01}-J_{23}-J_{45}-J_{67} \\
& X_{2}=J_{02}-J_{13}-J_{46}-J_{57} \\
& X_{3}=J_{03}-J_{12}-J_{47}-J_{56} \\
& X_{4}=J_{04}-J_{15}-J_{26}-J_{37} \\
& X_{5}=J_{05}-J_{14}-J_{27}-J_{36} \\
& X_{6}=J_{06}-J_{17}-J_{24}-J_{35} \\
& X_{7}=J_{07}-J_{16}-J_{26}-J_{34}
\end{aligned}
$$

which may be transported to form a basis of smooth nonvanishing leftinvariant vector fields on the seven-sphere, given the embedding of $S^{7}$ into $S O(8)$ [15]. The Killing vector fields on a coset space $G / H$ represent generators of the isometry group $G \times N(H) / H$. When $G$ is the maximal transitive group of a constant curvature space, it includes every isometry and would be a subgroup of the diffeomorphism group [16]. The exponential maps of the tangent vector fields on $S^{7}$ are elements in the isometry group $S O(8)$ and represent diffeomorphisms.

The homogeneous term is

$$
\omega\left(R_{g^{*}} \sigma_{*} \cdot \xi_{\mu}\right)=R_{\left(a_{y} g a_{y \cdot g}^{-1}\right)^{*}} \omega\left(\sigma_{*} \cdot \xi_{\mu}\right)
$$

Where $L_{y} y^{\prime}=y^{\prime} R_{a_{y}}$. It remains to beestablished if $h(y, g)=a_{y} g a_{y \cdot g}^{-1}$ is independent of $y$ for the $R_{g}^{T}=e^{t_{i} X_{i}}$.

Noting that

$$
\begin{aligned}
& h_{00}=1 \\
& h_{i 0}=0 i=1, \ldots, 7 \\
& h_{11}=\left(y_{0}^{2}+y_{1}^{2}\right)\left(c_{00} c_{11}-c_{10} c_{01}\right)+\left(y_{0} y_{3}+y_{1} y_{2}\right)\left(c_{00} c_{21}-c_{10} c_{31}-c_{20} c_{01}+c_{30} c_{11}\right) \\
& +\left(y_{0} y_{2}-y_{1} y_{3}\right)\left(-c_{00} c_{31}-c_{10} c_{21}+c_{20}+c_{01}\right) \\
& +\left(y_{0} y_{5}-y_{1} y_{4}\right)\left(-c_{00} c_{11}-c_{10} c_{51}+c_{40} c_{01}+c_{50} c_{11}\right) \\
& +\left(y_{0} y_{6}-y_{1} y_{7}\right)\left(-c_{00} c_{71}-c_{10} c_{41}+c_{60} c_{11}+c_{70} c_{01}\right)+\left(y_{2}^{2}+y_{3}^{2}\right)\left(-c_{20} c_{31}+c_{30} c_{21}\right) \\
& +\left(y_{3} y_{4}-y_{2} y_{5}\right)\left(-c_{20} c_{41}-c_{30} c_{51}+c_{40} c_{21}+c_{50} c_{31}\right) \\
& +\left(y_{2} y_{4}-y_{3} y_{5}\right)\left(-c_{20} c_{51}-c_{30} c_{41}+c_{40} c_{31}+c_{50} c_{21}\right) \\
& +\left(y_{3} y_{6}-y_{2} y_{7}\right)\left(-c_{20} c_{01}-c_{30} c_{11}+c_{60} c_{21}+c_{70} c_{31}\right) \\
& +\left(y_{3} y_{7}-y_{2} y_{6}\right)\left(-c_{20} c_{71}-c_{30} c_{61}+c_{60} c_{31}+c_{70} c_{21}\right) \\
& +\left(y_{4}^{2}+y_{5}^{2}\right)\left(-c_{40} c_{51}+c_{50} c_{41}\right)+\left(y_{5} y_{6}-y_{4} y_{7}\right)\left(-c_{40} c_{61}-c_{50} c_{71}+c_{60} c_{41}+c_{70} c_{51}\right)
\end{aligned}
$$




$$
\begin{aligned}
& \quad+\left(y_{5} y_{7}+y_{4} y_{6}\right)\left(-c_{40} c_{71}+c_{50} c_{61}-c_{60} c_{51}+c_{70} c_{41}\right)+\left(y_{6}^{2}+y_{7}^{2}\right)\left(-c_{60} c_{71}+c_{70} c_{61}\right) \\
& -\left(\left(c_{i 0}, c_{j 1}\right) \leftrightarrow\left(c_{i 2}, c_{j 3}\right)+\left(c_{i 0}, c_{j 1}\right) \leftrightarrow\left(c_{i 4}, c_{j 5}\right)+\left(c_{i 0}, c_{j 1}\right)\left(c_{i 6}, c_{j 7}\right)\right) \\
& \vdots
\end{aligned}
$$

There are 15 conditions from the $y$-independence of $h_{11}$. Given an equal number of each of the elements $h_{i j}, i, j=, \ldots, 7$, the maximum number of equations for the coefficients of the matrix representing $g$ is $(64)(21)=1344$. Although this number may be reduced by $\mathrm{SO}(8)$ relations and redundancies, the system will be overdetermined with no nontrivial solutions.

For $S^{3}$, left and right multiplication by the group commute. The analogous identity does not hold for $S^{7}$ and $\mathrm{SO}(8)$ matrices.

$$
\begin{aligned}
& \tilde{g}^{T} l_{L}(y)^{T} \tilde{G}\left[\iota_{L}(y \cdot \tilde{g})^{-1}\right]^{T}=\tilde{g}^{T} l_{L}(y)^{T} \tilde{g}\left(\iota_{L}\left(\tilde{g}^{-1}\right)\left(l_{L}\left(y^{-1}\right)\right)^{T}\right. \\
& =\tilde{g}^{T} l_{L}(y)^{T} \tilde{g} l_{L}\left(y^{-1}\right)^{T} \iota_{L}\left(\tilde{g}^{-1}\right)^{T} \\
& \leftrightarrow \tilde{g}^{-1} y^{-1}\left[\left(y \cdot \tilde{g}^{\prime}\right) \tilde{g}\right] \\
& \neq \tilde{g}^{-1}\left(y^{-1}\left(y \cdot \tilde{g}^{\prime}\right) \tilde{g}\right) \\
& =\tilde{g}^{-1}\left(\tilde{g}^{\prime} \cdot \tilde{g}\right)
\end{aligned}
$$

Then the matrix $h(y, g)$ will be $y$-dependent unless the coefficients $c_{i j}$ satisfy a set of relations to remove the fibre coordinate from $a_{y} g a_{y \cdot g}^{-1}$ given the equation $y_{0}^{2}+y_{1}^{2}+y_{2}^{2}+y_{3}^{2}+y_{4}^{2}+y_{5}^{2}+y_{6}^{2}+y_{7}^{2}=1$.

\section{Proposition 2.2}

The decomposition of the adjoint representation of SU(4) into the adjoint, fundamental and trivial representations of $\mathrm{SU}(3)$ includes a single one-dimensional factor. The set of conditions required for the independence of the inhomogeneous term in the connection transformation rule is given. The homogeneous term has no effect on the remaining component of the tangent vector to the fibre and the $\mathrm{U}(1)$ gauge symmetry.

Proof: The decomposition of the adjoint representation of $\mathrm{SU}(4)$ is $\underline{15} \rightarrow \underline{8} \oplus \underline{3} \oplus \underline{\overline{3}} \oplus \underline{1}$. There is a $\mathrm{U}(1)$ gauge invariance in four dimensions when the initial symmetry is $\mathrm{SU}(4)$ [10].

The isometry group of $S U(4) / S U(3)$ is $S U(4) / N(S U(3)) / S U(3)$, and the dimension can be increased to the degree of symmetry of $S^{7}$ with the round-sphere metric invariant under $S U(8)$ [17]. Even though $N(S U(3)) / S U(3) \simeq U(1)$ [18], this $U(1)$ factor is not required in a direct comparison with the reduction of the adjoint representation of $S U(4)$. The group $S U(4)$ can be embedded in $S O(8)$, and therefore, it forms a subgroup of the diffeomorphism group of the sphere. With an Euler angle parameterization, let $y=\left(y_{0}+i y_{1} y_{2}+i y_{3} y_{4}+i y_{5} y_{6}+i y_{7}\right)$ and $J_{A}$ be the generators of $\mathrm{SU}(4)$ :

$$
\begin{aligned}
& J_{1}=\left(\begin{array}{cccc}
0 & 1 & 0 & 0 \\
-1 & 0 & 0 & 0 \\
0 & 0 & 0 & 0 \\
0 & 0 & 0 & 0
\end{array}\right) J_{2}=\left(\begin{array}{cccc}
0 & 0 & 1 & 0 \\
0 & 0 & 0 & 0 \\
-1 & 0 & 0 & 0 \\
0 & 0 & 0 & 0
\end{array}\right) J_{3}=\left(\begin{array}{cccc}
0 & 0 & 0 & 1 \\
0 & 0 & 0 & 0 \\
0 & 0 & 0 & 0 \\
-1 & 0 & 0 & 0
\end{array}\right) \\
& J_{4}=\left(\begin{array}{llll}
0 & i & 0 & 0 \\
i & 0 & 0 & 0 \\
0 & 0 & 0 & 0 \\
0 & 0 & 0 & 0
\end{array}\right) J_{5}=\left(\begin{array}{llll}
0 & 0 & i & 0 \\
0 & 0 & 0 & 0 \\
i & 0 & 0 & 0 \\
0 & 0 & 0 & 0 \\
& &
\end{array}\right) J_{6}=\left(\begin{array}{llll}
0 & 0 & 0 & i \\
0 & 0 & 0 & 0 \\
0 & 0 & 0 & 0 \\
i & 0 & 0 & 0 \\
& & &
\end{array}\right)
\end{aligned}
$$

$$
\begin{aligned}
& J_{7}=\left(\begin{array}{cccc}
i & 0 & 0 & 0 \\
0 & -i & 0 & 0 \\
0 & 0 & 0 & 0 \\
0 & 0 & 0 & 0
\end{array}\right) \quad J_{8}=\left(\begin{array}{cccc}
0 & 0 & 0 & 0 \\
0 & 0 & 1 & 0 \\
0 & -1 & 0 & 0 \\
0 & 0 & 0 & 0
\end{array}\right) \quad J_{9}=\left(\begin{array}{cccc}
0 & 0 & 0 & 0 \\
0 & 0 & i & 0 \\
0 & i & 0 & 0 \\
0 & 0 & 0 & 0
\end{array}\right) \\
& J_{10}=\left(\begin{array}{cccc}
0 & 0 & 0 & 0 \\
0 & 0 & 0 & 1 \\
0 & 0 & 0 & 0 \\
0 & -1 & 0 & 0
\end{array}\right) \quad J_{11}=\left(\begin{array}{cccc}
0 & 0 & 0 & 0 \\
0 & 0 & 0 & i \\
0 & 0 & 0 & 0 \\
0 & i & 0 & 0
\end{array}\right) \quad J_{12}=\left(\begin{array}{cccc}
0 & 0 & 0 & 0 \\
0 & 0 & 0 & 0 \\
0 & 0 & 0 & 1 \\
0 & 0 & -1 & 0
\end{array}\right) \\
& J_{13}=\left(\begin{array}{cccc}
0 & 0 & 0 & 0 \\
0 & 0 & 0 & 0 \\
0 & 0 & 0 & i \\
0 & 0 & -i & 0
\end{array}\right) \quad J_{14}=\left(\begin{array}{cccc}
0 & 0 & 0 & 0 \\
0 & i & 0 & 0 \\
0 & 0 & -i & 0 \\
0 & 0 & 0 & 0
\end{array}\right) \quad J_{15}=\left(\begin{array}{cccc}
0 & 0 & 0 & 0 \\
0 & 0 & 0 & 0 \\
0 & 0 & i & 0 \\
0 & 0 & 0 & -i
\end{array}\right)
\end{aligned}
$$

and

$$
\begin{aligned}
& y\left(d_{A} J_{A}\right)\left(l_{L}\left(y^{-1}\right)\right)^{T}=\left(\begin{array}{lllllll}
\cos \theta_{2} & \cos & \theta_{1} & \cos & \phi_{1} & e^{i \delta_{1}} & -\sin \theta_{1} e^{i\left(\delta_{1}-\sigma_{3}\right)}
\end{array}\right. \\
& \left.-\cos \theta_{2} \sin \theta_{1} e^{i \delta_{1}}-\cos \theta_{2} \cos \theta_{1} \sin \phi_{1} e^{i\left(\delta_{1}-\sigma_{1}\right)}\right) \\
& \left(\begin{array}{cccc}
i d_{7} & d_{1}+i d_{4} & d_{2}+i d_{5} & d_{3}+i d_{6} \\
-d_{1}+i d_{4} & -i d_{7}+i d_{14} & d_{8}+i d_{9} & d_{10}+i d_{11} \\
-d_{2}+i d_{5} & -d_{8}+i d_{9} & -i d_{14}+i d_{15} & d_{12}+i d_{13} \\
-d_{3}+i d_{6} & -d_{10}+i d_{11} & -d_{12}+i d_{13} & -i d_{15}
\end{array}\right) \\
& \left(\begin{array}{cccc}
\cos \theta_{2} \cos \theta_{1} \cos \phi_{1} e^{-i \delta_{1}} & \sin \theta_{2} \cos \theta_{1} \cos \phi_{1} e^{-i \sigma_{2}} & \sin \theta_{1} \cos \phi_{1} e^{-i \sigma_{2}} & \sin \phi_{1} e^{i\left(\delta_{1}-\sigma_{1}\right)} \\
-\sin \theta_{2} e^{-i\left(\delta_{1}-\sigma_{3}\right)} & \cos \theta_{2} & 0 & 0 \\
-\cos \theta_{2} \sin \theta_{1} e^{-i\left(\delta_{1}-\sigma_{3}\right)} & -\sin \theta_{2} \sin \theta_{1} e^{i\left(\sigma_{2}-\sigma_{3}\right)} & \cos \theta_{1} & 0 \\
-\cos \theta_{2} \cos \theta_{1} \sin \phi_{1} e^{-i\left(\delta_{1}-\sigma_{1}\right)} & -\sin \theta_{2} \cos \theta_{1} \sin \phi_{1} e^{i\left(\sigma_{1}-\sigma_{3}\right)} & -\sin \theta_{1} \sin \phi_{1} e^{i\left(\sigma_{1}-\sigma_{2}\right)} & \cos \phi_{1} e^{i \delta_{1}}
\end{array}\right)
\end{aligned}
$$

Denoting this vector by $\left(c_{0} c_{1} c_{2} c_{3}\right)$, independence of $c_{0}$ with respect to $y$ requires

$$
i d_{7}=-i d_{7}+i d_{14}=-i d_{14}+i d_{15}=-i d_{15}
$$

and

$$
\begin{aligned}
& \sigma_{1}=\sigma_{2}=\sigma_{3}=0 \\
& d_{4}=d_{5}=d_{6}=d_{9}=d_{11}=d_{13}=0
\end{aligned}
$$

or similar combinations

$$
\begin{aligned}
& \sigma_{1}=\pi 2 \quad \sigma_{2}=\sigma_{3}=0 \\
& d_{3}=d_{4}=d_{5}=d_{9}=d_{10}=d_{12}=0 ; \\
& \sigma_{1}=0 \quad \sigma_{2}=\frac{\pi}{2} \quad \sigma_{3}=0 \\
& D_{2}=d_{4}=d_{6}=d_{8}=d_{11}=d_{12}=0 ; \\
& \sigma_{1}=\sigma_{2}=0 \quad \sigma_{3}=\frac{\pi}{2} \\
& d_{1}=d_{5}=d_{6}=d_{9}=d_{10}=d_{13}=0 ; \\
& \sigma_{1}=\sigma_{2}=\frac{\pi}{2} \quad \sigma_{3}=0 \\
& d_{2}=d_{3}=d_{4}=d_{8}=d_{10}=d_{13}=0 ; \\
& \sigma_{1}=\pi 2 \quad \sigma_{2}=0 \quad \sigma_{3}=\frac{\pi}{2}
\end{aligned}
$$




$$
\begin{aligned}
& d_{1}=d_{3}=d_{5}=d_{8}=d_{11}=d_{12}=0 ; \\
& \sigma_{1}=0 \quad \sigma_{2}=\sigma_{3}=\frac{\pi}{2} \\
& d_{1}=d_{2}=d_{6}=d_{9}=d_{10}=d_{12}=0 ; \\
& \sigma_{1}=\sigma_{2}=\sigma_{3}=\frac{\pi}{2} \\
& D_{1}=d_{2}=d_{3}=d_{9}=d_{11}=d_{13}=0 .
\end{aligned}
$$

Consider the parameters which satisfy Eqs.(2.9) and (2.10). Then

$$
c_{1}=d_{1} \cos \theta_{1} \cos \phi_{1} e^{i \delta_{1}}+d_{8} \sin \theta_{1} e^{i \delta_{1}}+d_{10} \cos \theta_{1} \sin \phi_{1} e^{i \delta_{1}}
$$

which is independent of $y$ if any of the following conditions hold:

$$
\begin{aligned}
& d_{1}=d_{8}=d_{10}=0 \\
& \theta_{1}=\pi 2 d_{8}=0 \\
& \varphi_{1}=\pi 2 d_{8}=0 \quad d_{10}=0 \\
& d_{1}=0 \quad \theta_{1}=0 \quad d_{10}=0 \\
& d_{1}=0 \quad d_{8}=0 \quad \varphi_{1}=0 \\
& d_{1}=0 \quad \theta_{1}=0 \quad \varphi_{1}=0 \\
& \varphi_{1}=\pi 2 \quad \theta_{1}=0 d_{10}=0
\end{aligned}
$$

Suppose that the first set of conditions are valid. It follows that $c_{2}=d_{2} \cos \theta_{2} \cos \phi_{1} e^{i \delta_{1}}+d_{12} \cos \theta_{2} \sin \phi_{1} e^{i \delta_{1}}$

Again, $c_{2}$ is independent of $y$ if

$$
\begin{aligned}
& d_{2}=d_{12}=0 \\
& d_{2}=0 \varphi_{1}=0 \\
& \phi_{1}=\frac{\pi}{2} \quad d_{12}=0 \\
& \theta_{2}=\pi 2 .
\end{aligned}
$$

With the first choice,

$$
c_{3}=d_{3} \cos \theta_{2} \cos \theta_{1} e^{i \delta_{1}} \text {. }
$$

Then

$$
\begin{aligned}
& D_{3}=0 \\
& \theta_{1}=\frac{\pi}{2} \\
& \theta_{2}=\frac{\pi}{2} \\
& \theta_{1}=\theta_{10} \theta_{2}=\theta_{20} \delta_{1}=\delta_{10} .
\end{aligned}
$$

Since $c_{3}$ would vanish for the first three conditions, there would be no inhomogeneous term in the gauge transformation. Given the last set of relations, $c_{3}=d_{3} \cos \theta_{20} \cos \theta_{10} e^{2 i \delta_{10}}$.

$$
\begin{aligned}
& \text { If } \theta_{1}=\frac{\pi}{2} \text { and } d_{8}=0 \text {, } \\
& c_{2}=\left[\left(y_{0}+i y_{1}\right)\left(i d_{7}\right)+\left(y_{2}+i y_{3}\right)\left(-d_{1}+i d_{4}\right)+\left(y_{1}+i y_{5}\right)\left(-d_{2}+i d_{5}\right)\right. \\
& \left.+\left(y_{6}+i y_{7}\right)\left(-d_{3}+i d_{6}\right)\right] \sin \theta_{1} \cos \phi_{1} e^{-i \sigma_{2}} \\
& +\left[\left(y_{0}+i y_{1}\right)\left(d_{2}+i d_{5}\right)+\left(y_{2}+i y_{3}\right)\left(d_{8}+i d_{9}\right)+\left(y_{4}+i y_{5}\right)\left(-d_{14}+i d_{15}\right)\right. \\
& \left.+\left(y_{6}+i y_{7}\right)\left(-d_{12}+i d_{13}\right)\right] \cos \theta_{1} \\
& +\left[\left(y_{0}+i y_{1}\right)\left(d_{3}+i d_{6}\right)+\left(y_{2}+i y_{3}\right)\left(d_{10}+i d_{11}\right)+\left(y_{4}+i y_{5}\right)\left(d_{12}+i d_{13}\right)\right. \\
& \left.+\left(y_{6}+i y_{7}\right)\left(-d_{15}\right)\right]\left(-\sin \theta_{1} \sin \phi_{1} e^{-i \sigma_{2}}\right) \\
& =d_{1} \sin \theta_{2} \cos \phi_{1} e^{i \delta_{1}}+d_{2} \cos \phi_{1} \cos \theta_{2} e^{i \delta_{1}}
\end{aligned}
$$

$$
-d_{10} \sin \theta_{2} \quad e^{i \delta_{1}}+d_{12} \cos \quad \theta_{2} \quad e^{i \delta_{1}},
$$

which equals zero if $d_{1}=d_{2}=d_{10}=d_{12}=0$ or $\theta_{2}=\frac{\pi}{2}, \phi_{1}=\frac{\pi}{2}$ and $d_{10}=0$. In the first instance, $c_{3}=0$, and there is no gauge transformation. If two of the angles are fixed, $c_{3}=d_{1} e^{2 i \delta_{1}}$. Setting $\delta_{1}=0$ leaves no remaining $U(1)$ gauge invariance.

With the choice $\phi_{1}=\frac{\pi}{2}, d_{8}=0, d_{10}=0$

$$
c_{2}=-d_{8} \sin \theta_{1} \cos \theta_{2} e^{i \delta_{1}}+d_{10} \sin \theta_{1} \sin \theta_{2} e^{i \delta_{1}}+i d_{12} \cos \theta_{2} e^{i \delta_{1}}
$$

Is independent of $y$ if $d_{12}=0$. There remain three nonvanishing group parameters $d_{1}, d_{2}$ and $d_{3}$. It follows that

$$
\begin{aligned}
& c_{3}=\left[\left(y_{0}+i y_{1}\right)\left(i d_{7}\right)+\left(y_{2}+i y_{3}\right)\left(-d_{1}+i d_{4}\right)+\left(y_{4}+i y_{5}\right)\left(-d_{2}+i d_{5}\right)+\left(y_{6}+i y_{7}\right)(-\right. \\
& \left.\left.d_{3}+i d_{6}\right)\right] \\
& \quad \sin \phi_{1} e^{i\left(\delta_{1}-\sigma_{1}\right)} \\
& +\left[\left(y_{0}+i y_{1}\right)\left(d_{6}+i d 6\right)+\left(y_{2}+i y_{3}\right)\left(d_{10}+i d_{11}\right)+\left(y_{4}+i y_{5}\right)\left(d_{12}+i d_{13}\right)+\left(y_{6}+i y_{7}\right)\right. \\
& \left.\left(-d_{15}\right)\right] \\
& \quad \cos \phi_{1} e^{i \delta_{1}} \\
& \text { When } \phi_{1}=\frac{\pi}{2} \text { and } d_{8}=d_{10}=0, \\
& c_{3}=d_{1} \quad \sin \theta_{2} e^{i \delta_{1}}+d_{2} \quad \sin \theta_{1} \cos \theta_{2} e^{2 i \delta_{1}} \\
& +d_{3} \cos \theta_{1} \cos \theta_{2} e^{2 i \delta_{1}} .
\end{aligned}
$$

If $\theta_{1}=\theta_{2}=\delta_{1}=0, c_{3}=d_{3}$. When $\theta_{1}=\frac{\pi}{2}, \theta_{2}=0$ and $\theta_{2}=0, c_{3}=d_{2}$. However, since $\varphi_{1}$ is fixed, there is no $U(1)$ invariance remaining. Setting $d_{3}=0$, $\theta_{2}=\frac{\pi}{2}$ and $\delta_{1}=0, c_{3}=d_{1}$. The coefficient $d_{2}$ has not been set equal to zero, and yet, there is no angular variable for a second $U(1)$ symmetry.

If $d_{1}=0, \theta_{1}=0$ and $d_{10}=0$, the remaining group parameters are $d_{2}, d_{3}$ $d_{8}$ and $d_{12}$. Then

$$
c_{2}=d_{2} \cos \theta_{2} \cos \phi_{1} e^{i \delta_{1}}-d_{8} \sin \theta_{2} e^{i \delta_{1}}+d_{12} \cos \theta_{2} e^{i \delta_{1}}
$$

and $c_{2}=0$ if $d_{2}=d_{8}=d_{12}=0$ or $\theta_{2}=0$ and $d_{8}=0$. When $d_{3}$ is the only nonzero coefficient, $c_{3}=d_{3} \cos \theta_{2}\left(\sin \phi_{1}+\cos \phi_{1}\right) e^{i \delta_{1}}$, which equals $d_{3}$ if $\theta_{2}=0, \varphi_{1}=0$ or $\phi_{1}=\frac{\pi}{2}$ and $\delta_{1}=0$, leaving no Euler angles for a gauge transformation. The coordinate $c_{3}$ vanishes for $\theta_{2}=\frac{\pi}{2}$.

Suppose that $d_{1}=d_{8}=0$ and $\varphi_{1}=0$. The remaining group parameters are $d_{2}, d_{3}, d_{10}$ and $d_{12}$. Then $c_{2}=d_{2} \sin ^{2} \theta_{1} \cos \theta_{2} \cdot e^{i \delta_{1}}$ $-\mathrm{d}_{10} \sin \theta^{1} \sin \theta^{2} e^{i \delta_{1}}$, which is zero if $d_{2}=d_{10}=0$ or $\theta_{1}=0$. When $d_{2}=d_{10}=0$, $\mathrm{c}_{3}=\mathrm{d}_{3} \cos \theta_{1} \cos \theta_{2} e^{i \delta_{1}}-\mathrm{d} 12 \sin \theta_{1} \cos \theta_{2} e^{i \delta_{1}}$. If $\theta_{1}=\theta_{2}=\delta_{1}=0, c_{3}=d_{3}$, while the Eulerangles $\theta_{1}=\frac{\pi}{2}, \theta_{2}=0, \delta_{1}=0$ yield $c_{3}=d_{12}$. Although there are two non vanishing coefficients, there are no free anguler variables.

If $\mathrm{d}_{1}=0, \theta_{1}=0$ and $\varphi_{1}=0, c_{2}=-d_{8} \sin \theta_{2} e^{i \delta_{1}}+d_{12} \cos \theta_{2} e^{i \delta_{1}}$. Let $d_{8}=0$, such that $c_{3}=d_{3} \cos \theta_{2} e^{2 i \delta_{1}}-d_{10} \sin \theta_{2} e^{2 i \delta_{1}}$. With $\theta_{2}=0$ and $\delta_{1}=0$, $c_{3}=d_{3}$, while $c_{3}=-d_{10}$ if $\theta_{2}=\frac{\pi}{2}$ and $\theta_{1}=0$. There are two non - zero coefficients and all of the angular variables have fixed values.

Finally, when $\phi_{1}=\frac{\pi}{2}, \theta_{1}=0$ and $d_{10}=0, c_{2}=-d_{8} \sin \theta_{2} e^{i \delta_{1}}+d_{12} \cos \theta_{2} e^{i \delta_{1}}$. Setting $d_{8}=d_{12}=0, c_{3}=d_{1} \sin \theta_{2} e^{i \delta_{1}}+d_{3} \cos \theta_{2} e^{i \delta_{1}}$. If $\theta_{2}=0$ or $\theta_{2}=\frac{\pi}{2}$ and, $\delta_{1}=0, c_{3}$ is non vanishinh and independent of the Eulerangles. Howevr, these variables and fixed and there is no freedom for a U(1) gauge transformation. 
If the parameters of the matrix $\iota_{L}\left(y^{-1}\right)^{T}$ satisfy any set of conditions in Eq.(2.11), the same numbers of coefficients $d_{A}$ and phases $\sigma_{i}$ are eliminated. Therefore, $c_{1}$ has a similar form with different coefficients. The set of variables fixed by the independence of $c_{1}, c_{2}$ and $c_{3}$ will be determined by the identical method.

Returning to the initial choice of values of the coefficients, independence of the homogeneous term follows from the equality of $L_{y}^{T} R_{g}^{T} L_{(y \cdot g)^{-1}}^{T}=\iota_{L}(\mathrm{y})^{T} \exp \left(d_{3} J_{3}\right)\left[\imath(y \cdot g)^{-1}\right]^{T}$ with the identity, which follows from the unitarity derived from

$$
\left(\begin{array}{cccc}
\cos \theta_{20} \cos \theta_{10} \cos \left(\phi_{1}-d_{3}\right) & -\sin \theta_{20} & -\cos \theta_{20} \sin \theta_{10} & -\cos \theta_{20} \cos \theta_{10} \sin \left(\phi_{1}-d_{3}\right) \\
\sin \theta_{20} \cos \theta_{10} \cos \left(\phi_{1}-d_{3}\right) & \cos \theta_{20} & \cos \theta_{10} & -\sin \theta_{10} \sin \left(\phi_{1}-d_{3}\right) \\
\sin \theta_{10} \cos \left(\phi_{1}-d_{3}\right) & 0 & \cos \theta_{10} & -\sin \theta_{10} \sin \left(\phi_{1}-d_{3}\right) \\
\sin \left(\phi_{1}-d_{3}\right) & 0 & 0 & \cos \left(\phi_{1}-d_{3}\right)
\end{array}\right)
$$

The transformation rule of the gauge potential would be

$$
A_{\mu} \rightarrow A_{\mu}+\partial_{\mu} d_{3}
$$

which represents a $\mathrm{U}(1)$ gauge invariance.

The new feature of these calculations is the existence of a different residual gauge group given the same topology for the compact space inducing the dimensional reduction. It may be noted that $\mathrm{SU}(4) / \mathrm{SU}(3)$ is a $\mathrm{U}(1)$ bundle over $C P_{3}$. The number of $\mathrm{U}(1)$ factors in the fibre of the bundle $G \rightarrow S / R \rightarrow B$ would determine the remaining abelian group invariance.

The isomorphism between the residual gauge symmetry resulting from reduction over a coset space and the invariance group derived from the reduction of the generalized transformation rule of the connection form, induced by the action of elements in the isometry group, to the base space will be proven.

\section{Theorem 2.3}

The residual symmetry group in generalized dimensional reduction over a coset space $S / R$ coincides the the gauge group preserving the invariance of an action constructed from a connection form for a bundle $E(M, S, S / R, \pi)$.

Proof: The centralizer of $R$ in $S, \mathrm{C}(\mathrm{R})$, determines the number of $\mathrm{R}$-singlets in the decomposition of the adjoint representation of $\mathrm{S}$ and the gauge group of the reduced theory [10]. The $y$-independence of the terms in the generalized connection transformation rule depends on that of $L_{(y \cdot g)^{*}}^{-1} R_{g^{*}} L_{y^{*}}$. When left on $S / R$ and right multiplication on $R$ within the group commute,

$$
L_{y \cdot g}^{-1} R_{g} L_{y}=L_{y \cdot g}^{-1} L_{y} R_{g}=L_{g}^{-1} L_{y}^{-1} L_{y} R_{g} .
$$

Therefore, the group elements in the centralizer would belong to the subgroup of the isometry group yielding a transformation rule independent of the fibre coordinate. Although inclusion in the centralizer is a sufficient for the gauge potential to be defined in the base space. The inhomogeneous term is the tangent mapping of a transformation which can be represented as

$$
\begin{aligned}
& y \cdot \exp \left(d_{A B} J_{A B}\right)^{T} \cdot d_{C D} J_{C D} l_{L}\left(\left(y \cdot \exp \left(d_{E F} J_{E F}\right)\right)^{-1}\right)^{T} \\
& =y\left(\exp \left(-d_{A B} J A B\right)\right) d_{C D} J_{C D} l_{L}\left(\exp \left(d_{E F} J_{E F}\right)\right) \iota_{L}\left(y^{-1}\right)^{T} .
\end{aligned}
$$

When $J_{A B}$ belongs to the Lie algebra of the centralizer of $R$ in $S$, the matrix representing $y$ can be commuted through the linear combinator of generators to give

$$
\exp \left(-d_{A B} J_{A B}\right) d_{C D} J_{C D} t_{L}\left(\exp \left(d_{E F} J_{E F}\right)\right)
$$

which is independent of $y$. The $y$-dependence can be removed from this matrix product only if these matrices commute. Therefore, the reduction of the symmetry group to the centralizer $C(R)$ is necessary for gauge transformation to be defined in the base space. The residual gauge symmetry is identical with either method.

An exceptional fibre bundle $E\left(M, \operatorname{Diff}\left(S^{1}\right), S^{1}\right)$ has been found to satisfy the conditions for a factorization of the fibre-coordinate dependence from the transformation rule for the connection form [19]. Since the normalizer of $U(1)$ in $\operatorname{Diff}\left(S^{1}\right)$, the decomposition will yield an infinite number of independent generators of the residual gauge group $U(1) \times \ldots \times U(1)$.

\section{Parallelizability and Dimensional Reduction}

The dimensional reduction of bosonic field theories over a coset manifold and the Lagrangian in four dimensions have been described for various gauge groups. The bosonic sector of the Weinberg-Salam model is derived by dimensional reduction of the six-dimensional YangMills action over $S^{2}$. This model cannot be extended to include fermions because the expansion of fermion fields in generalized spherical harmonics with half-integer indices is prevented by the conditions on the gauge transformations derived from rotational invariance [20].

Supersymmetric Yang-Mills theory in six dimensions, therefore, cannot be consistently dimensionally reduced over $S^{2}$. This result reflects the absence of a dimensional reduction of supersymmetric theories over manifolds that are not parallelizable. By contrast with the bosonic field, the fermion field requires a spin bundle which is defined as the square root of the frame bundle on a curved manifold. The field $\psi_{\mu}=e_{m} u^{a} \psi_{a}$, where $e_{\mu}^{a}$ is a vielbein on the manifold and $\psi_{a}$ forms a representation of the spin covering of the tangent space group, exists only if the vielbein is smooth and nonvanishing. These properties of the frame of orthonormal tangent vectors require a global parallelism. A similar conclusion is found for spinors in four dimensions, which are defined if the second Stiefel-Whitney class vanishes [21].

The commutation relations of Killing vectors generating the isometry group of a coset manifold will remain consistent even though these vectors, which may be expanded in terms of an orthonormal basis, vanish at some point on this space. The Killing vectors only generate motion along the fibres, which is projected to the base space, and the reduced bosonic fields are required to form representations of the isometry group. The anticommutators of supercharges in a supersymmetric theory also would consist of generators of the isometry group. However, the supercharges transform bosonic fields to fermionic fields, and the vanishing of the lift of the tangent vector on the base space affects the existence of this transformation in the total space of the bundle. Consequently, supersymmetric theories must be dimensionally reduced over parallelizable spaces.

The reduction of higher-dimensional fields over coset manifolds has been formulated for supergravity [10]. The decomposition of $S$ with respect to $R$ is sufficient to determine the residual gauge symmetries for the bosonic sector since it is determined by the image of the initial gauge group in the invariance group of the tangent bundle. Since the fermionic sector would transform under the same group, the gauge symmetries of the entire set of fields are established. Not all of the supersymmetries have to be broken, and a supersymmetric theory may be derived under this generalized dimensional reduction. The singlet representations of $R$ transform under the action of a $U(1)$ subgroup, and therefore, the corresponding bundle has a parallelizable fibre. More 
generally, the centralizer $C(R)$ would have to be cast in the form of an isometry group of a parallelizable submanifold of the coset space. Then the dimensional reduction procedure will be consistently yield a Lagrangian in four dimensions.

The existence of a fibre bundle geometry for the phenomenological theory based on a projection of the vector fields on $S^{7}$ to $S^{3} \times S^{3}$ follows from the uniqueness of the lifting of the parallelism on the group submanifold to one higher dimension [22]. A Lagrangian for this model may be given by the reduction of an octonionic Chern-Simons theory to an $S U(2) \times S U(2)$-invariant action, since the field equations are solved by flat connections required for a global parallelism on the internal symmetry space. The existence of a transformation from the $\mathrm{SU}(3)$ generators to octonions, where $\frac{\sqrt{3}}{2} \lambda_{8}, i \lambda_{1}, i \lambda_{2}, i \lambda_{3}, \frac{i}{2} \lambda_{5}, \frac{i}{2} \lambda_{5}$, $-\frac{i}{2} \lambda_{6}$ and $-\frac{i}{2} \lambda_{7}$ identified with $O_{A}, \stackrel{2}{=}=0, \ldots, 7$, yields a generic gauge transformation $g=e^{i \alpha_{A} o_{A}}$ [23]. The restriction to unit octonions would required $\sum_{A=0}^{7} \alpha_{A}^{2}=1$. Closure of the algebra of SU(3) gauge transformations with structure constants is no longer valid for $S^{7}$. The action of $S U(3)$ would preserve the inner product of the octonions since it is included in the automorphism group $G_{2}$. Consider the integral in an infinitesimal neighbourhood of a fixed point $y_{0}$

$$
\begin{aligned}
& I_{C S_{S_{y_{0}}}^{0}}=\int d^{3} x S C(A \wedge d A+23 A \wedge A \wedge A) \\
& \left.=\int^{y^{3}} x \varepsilon^{\mu v \rho} \operatorname{Sc}\left(A_{\mu} * \partial_{\nu} A_{\rho}\right)-23 f_{A B C} A_{v}^{A} A_{\rho}^{B} A_{\sigma}^{C}\right]
\end{aligned}
$$

Where $\operatorname{Sc}\left(\sum_{A} x_{A} O_{A}\right)=x_{0}, \quad A=\sum_{A} A_{A} O_{A}$ and $\sum_{A} A_{A}^{2}=1$, given that $\operatorname{Sc}\left(O_{A} O_{B} O_{C}\right)=-f_{A B C}, A, B, C=1, . ., 7$, which is independent of the order of the multiplication, even though $\left\{O_{A}\right\}$ is a basis for the octonions, since $f_{A B C}$ is antisymmetric. Under the transformation

$$
\begin{aligned}
& A_{\mu} \rightarrow A_{\mu}^{-1}+{ }^{-1} \partial_{\mu} \\
= & 1+\varepsilon_{A} O_{A} \\
& A_{\mu}^{A} O_{A} \rightarrow\left(1+\varepsilon_{A} O_{A}\right) A_{\mu}^{B} O_{B}\left(1-\varepsilon_{C} O_{C}\right) \partial_{\mu} \varepsilon_{D} O_{D}+O\left(\varepsilon^{2}\right) \\
= & A_{\mu}^{A} O_{A}-2 f_{A B C} \varepsilon_{A} A_{\mu}^{B} O^{C}+\partial_{\mu} \varepsilon_{A} O_{A}+O\left(\varepsilon^{2}\right) \\
= & A_{\mu}^{A} O_{A}-2 f_{A B C} \varepsilon_{A} A_{\mu}^{B} O^{C}+\partial_{\mu} \varepsilon_{A} O_{A}+O\left(\varepsilon^{2}\right)
\end{aligned}
$$

yielding the change in the components

$$
A_{\mu}^{A} \rightarrow A_{\mu}^{A}+\partial_{\mu} \varepsilon_{A}+2 f_{A B C} A_{\mu}^{B} \varepsilon_{C}
$$

Since the inverse of $O_{A}$ exists in a division algebra. The structure constants of the octonions do not satisfy the Jacobi identity and the variation of the integral will be

$$
\begin{aligned}
& { }_{S_{S_{y_{0}}}^{7}}^{0}=\int d^{3} x \varepsilon^{\mu \nu \rho}\left[\delta A_{\mu}^{0} \partial_{\nu} A_{\rho}^{0}+A_{\mu}^{0} \partial_{\nu} \delta A_{\rho}^{0}-\delta A_{\mu}^{1} \partial_{\nu} A_{\rho}^{1}-A_{\mu}^{1} \partial_{\nu} \delta A_{\rho}^{1}\right. \\
& -\ldots-\delta A_{\mu}^{7} \partial_{\nu} A_{\rho}^{7}-A_{\mu}^{7} \partial_{\nu} \delta A_{\rho}^{7} \\
& \left.-\frac{2}{3} f_{A B C}\left(\delta \mu^{A} A_{\nu}^{B} A_{\rho}^{C}+A_{\mu}^{A} \delta A_{\nu}^{B} A_{\rho}^{C}+A_{\mu}^{A} A_{\nu}^{B} \delta A_{\rho}^{C}\right)\right] \\
& =\int d^{3} x \varepsilon^{\mu \nu \rho}\left[\partial_{\mu} \varepsilon_{0} A_{\rho}^{0}+A_{\mu}^{0} \partial_{\nu} \partial_{\rho} \varepsilon_{0}-\sum_{=1}\left(\partial_{\mu} \varepsilon_{A} \partial_{\nu} A_{\rho}^{A}+A_{\mu}^{A} \partial_{\mu} \partial_{\nu} \varepsilon_{A}\right)\right. \\
& -2 \sum_{A, B, C=1}^{7}\left[f_{A B C} A_{\mu}^{B} \varepsilon_{C} \partial_{\nu} A_{\rho}^{A}+A_{\mu}^{A} \partial_{\mu}\left(f_{A B C} A_{\rho}^{B} \varepsilon^{C}\right)\right] \\
& -\frac{2}{3} \sum_{A, B, C=1}^{7} f_{A B C}\left[\left(\partial_{\mu} \varepsilon_{A}+\sum_{D, E=1}^{7} f_{A D E} A_{\mu}^{D} \varepsilon_{E}\right) A_{\nu}^{B} A_{\rho}^{C}\right.
\end{aligned}
$$

$$
\begin{aligned}
& +A_{\mu}^{A}\left(\partial_{\mu} \varepsilon_{B}+\sum_{D, E=1}^{7} f_{B D E} A_{v}^{D} \varepsilon_{E}\right) A_{\rho}^{C} \\
& \left.\left.+A_{\mu}^{B} A_{v}^{B}\left(\partial_{\rho} \varepsilon_{C}+\sum_{D, E=1}^{7} f_{C D E} A_{\rho}^{D} \varepsilon_{E}\right)\right]\right] \\
& =\int d^{3} x \varepsilon^{\mu \nu \rho}\left[S c\left(\partial_{\mu} \varepsilon^{*} \partial_{v} A_{\rho}\right)-2 \sum_{A, B, C=1}^{7} f_{A B C} A_{\mu}^{B} A_{\rho}^{B} \partial_{\nu} \varepsilon_{C}\right. \\
& -2 \sum_{A, B, C=1}^{7} f_{A B C} A_{\mu}^{A} A_{\nu}^{B} \partial_{\rho} \varepsilon_{C} \\
& -\frac{2}{3}\left(f_{A[B C} f_{|A| D] E} A_{\mu}^{D} A_{v}^{B} A_{\rho}^{C} \varepsilon_{E}+f_{[A|B| C]} f_{|B| D] E} A_{\mu}^{A} A_{v}^{D} A_{\rho}^{C} \varepsilon_{E}\right. \\
& \left.\left.+f_{[A B|C|} f_{|C| D] E} A_{\mu}^{A} A_{\mu}^{B} A_{\rho}^{D} \varepsilon_{E}\right)\right] \\
& =\int d^{3} x\left\{\partial_{\mu}\left(\varepsilon^{\mu v \rho} S c\left(\varepsilon^{*} \partial_{v} A_{\rho}\right)\right)-2 f_{A[B C} f_{|A| D] E} A_{\mu}^{D} A_{\nu}^{B} A_{\rho}^{C} \varepsilon_{E}\right\} .
\end{aligned}
$$

The nonvanishing of $f_{A[B C} f_{|A| D] E}$ is the difference from $\delta I_{C S}^{0}$ and the integral of a total derivative.

This Lagrangian will beinvariant underinfinitesimal transformations if $\left\{O^{A}\right\}$ is replaced by $\left\{\frac{2}{\sqrt{3}} O_{0},-i O_{1},-i O_{2},-i O_{3},-2 i O_{4},-2 i O_{5}, 2 i O_{6}, 2 i O_{7}\right\}$, representing the Gell-Mann matrices, and the structure constants fabc for $S U(3)$ are substituted for $f^{A B C}$. Then the Jacobi identity is valid and the gauge invariance of the transformed integral follows.

The combination $f_{A[B C} f_{A \mid D] E}$ may be identified with $-\phi_{B C D E}$, where

$\left[O_{A}, O_{B}, O_{C}\right]=2 \phi_{A B C D} O_{D}$. From the relations $f f \sim \phi, f \phi \sim f, \phi f \sim f$ and $\phi \phi \sim \phi$, the transformation and the Lagrangian may be modified to be $\delta A^{(1)} \sim \partial \varepsilon+f A \varepsilon+\phi A A \varepsilon$ and $L^{(1)} \sim \mathcal{E}(A d A+f A A A+\phi A A)$, such that $(\delta L)_{\text {int }}^{(1)} \sim f f A A A \varepsilon+f \varphi A A A A \varepsilon+\varphi f A A \varepsilon+\varphi \varphi A A A \varepsilon$. If there is a cancellation between $f A A A \varepsilon$ and $\phi \phi A A A \varepsilon$, there remains $f \phi A A A A \varepsilon$ and $\phi f A A \varepsilon$, yielding $f A A \varepsilon+f A A A A \varepsilon$. Introducing a infinite series of interaction terms, with

$$
L^{(2)} \sim \mathcal{E}\left[A d A+f \sum_{m=1}^{\infty} A^{2 m+1}+\varphi \sum_{n=1}^{\infty} A^{2 n}\right],
$$

the interaction terms in the variation would be

$$
\begin{aligned}
& \delta L^{(1)} \sim \mathcal{\varepsilon}[f A A \varepsilon+f A A A A \varepsilon+f A A A A A A \varepsilon+\ldots \\
& +\phi A A A \varepsilon+\phi A A A \varepsilon+\phi A A A A A \varepsilon+\phi A A A A A \varepsilon+\ldots]
\end{aligned}
$$

and there may be cancellations except for the first term $f A A \varepsilon$. Suppose that the term $\phi \varepsilon$ is included in transformation rule

$\delta A(2) \sim \partial \varepsilon+\phi \varepsilon+f A \varepsilon+\phi A A \varepsilon$.

Then

$$
\left.\delta L^{(2)}\right|_{\text {int }} \sim \varepsilon\left[f A A \varphi \varepsilon+\varphi A \varphi \varepsilon+f \sum_{m=2}^{\infty} A^{2 m} \varepsilon+\varphi \sum_{n=2}^{\infty} A^{2 n+1} \varepsilon\right]
$$

which reduces to $\phi A \varepsilon$ after the cancellation of the first term and the two series with the equivalent terms in Eq.(3.6). This term may be cancelled with the inclusion of $f A$ in the Lagrangian

$$
\begin{aligned}
& L^{(3)} \sim \mathcal{E}\left[A d A+f \sum_{m=0}^{\infty} A^{2 m+1}+\varphi \sum_{n=1}^{\infty} \varphi A^{2 n}\right] \\
& \text { Since }
\end{aligned}
$$

$$
\left.\delta(f A)\right|_{\text {int }} \sim f(f A \varepsilon+\varphi \varepsilon+\varphi A A \varepsilon) \sim \varphi A \varepsilon+f A A \varepsilon+f \varepsilon .
$$

The constant variation may be cancelled through the variation of an auxiliary field $\chi$, with $\delta \chi=\varepsilon$, in the Lagrangian

$$
L^{(4)} \sim \varepsilon\left[A d A+f \sum_{m=0}^{\infty} A^{2 m+1}+\varphi \sum_{n=1}^{\infty} \varphi A^{2 n}+f \chi\right] .
$$


The kinetic terms in the variation of the $L^{(1)}$ are

$\delta L^{(1)}{ }_{k i n} \sim \partial \varepsilon \partial A+A \partial \partial \varepsilon+f A \partial A \varepsilon+\phi A A \partial A \varepsilon+\phi A A A \partial \varepsilon+f A A \partial \varepsilon+\phi A \partial \varepsilon .(37)$

Cancellation of $f A A \varepsilon$ leaves $f A \partial A \varepsilon, \phi A A \partial A \varepsilon$ and $\phi A A A \partial \varepsilon$. The last term may be cancelled by the variation of $\delta(\phi A A A A)$ in $L^{(2)}$, while the other two terms require a change in the transformation rule

$$
\delta A \sim \partial \varepsilon+f \sum_{r=0}^{\infty} A^{2 r+1} \varepsilon+\varphi \sum_{s=1}^{\infty} A^{2 s} \varepsilon .
$$

Then the variation of the kinetic term in $L^{(1)}$ would yield also

$$
\varepsilon\left[f \sum_{r=0}^{\infty} A^{2 r+1} \partial A \varepsilon+\varphi \sum_{s=1}^{\infty} A^{2 s} \partial A \varepsilon\right]
$$

Similarly, in $L^{(2)}$,

$$
\begin{aligned}
& \left.\delta\left(f \sum_{m=1}^{\infty} A^{2 m+1}\right)\right|_{\partial \varepsilon} \sim f \sum_{m=1}^{\infty} A^{2 m} \partial \varepsilon \\
& \left.\delta\left(\varphi \sum_{n=1}^{\infty} A^{2 n}\right)\right|_{\partial \delta} \sim \varphi \sum_{n=1}^{\infty} A^{2 n-1} \varepsilon .
\end{aligned}
$$

The two sums in Eq.(3.14) can be written as

$$
\partial\left(f \sum_{r=1}^{\infty} A^{2 r} \varepsilon\right)-f \sum_{r=1}^{\infty} A^{2 r} \partial \varepsilon+\partial\left(\varphi \sum_{s=1}^{\infty} A^{2 s+1} \varepsilon\right)-\varphi \sum_{s=1}^{\infty} A^{2 s+1} \varepsilon
$$

Giving a total derivative and a cancellation with the sums in Eq.(3.15). The effect of the series in Eq.(3.13) on $f A$ and the sums in $L^{(4)}$ is

$$
\begin{aligned}
& f \sum_{s=0}^{\infty} A^{2 s} \varepsilon+\varphi \sum_{r=0}^{\infty} A^{2 r+1} \varepsilon+\varphi \sum_{m=1}^{\infty} \sum_{r=0}^{\infty} A^{2 m+2 r+1} \varepsilon \\
& +f \sum_{s=1}^{\infty} \sum_{s=0}^{\infty} A^{2 m+2 s} \varepsilon+f \sum_{n=1}^{\infty} \sum_{r=0}^{\infty} A^{2 n+2 r} \varepsilon+\varphi \sum_{n=1}^{\infty} \sum_{s=0}^{\infty} A^{2 n+2 s-1} \varepsilon .
\end{aligned}
$$

The series may cancel because the parity of the sums multiplying $f$ and $\phi$ is unchanged. Since $\left.\delta(f A)\right|_{\partial \varepsilon}=f_{\partial \varepsilon}$, it must be cancelled by the variation of $f \chi$. Then the Lagrangian would be

$$
L^{(5)} \sim \varepsilon\left[A d A+f \partial \chi+f \sum_{m=0}^{\infty} A^{2 m+1}+\varphi \sum_{n=1}^{\infty} \varphi A^{2 n}+f \chi\right] .
$$

Since $\varepsilon^{u v}$ has rank three, each term must have three tensor indices in the bracketed expression, which can be be arranged by including the normal vector $n^{\mu}$ to the three-dimensional boundary of a fourdimensional manifold and $n_{\mu}^{A}$, a vector in the total bundle, to contract fibre coordinate indices.

Under global transformations, the elements $O_{A}$ would be replaced by vector fields $V_{\alpha}, \alpha=1, \ldots, 7$ and the commutators give rise to structure functions on the sphere. Replacing the structure functions at a point by global vector fields, the interaction term can be replaced by:

$$
\frac{2}{3} \varepsilon^{\mu \nu \rho} A_{\mu}^{\alpha} A_{\nu}^{\beta} A_{\rho}^{\gamma}\left\langle\left[V_{\alpha}, V_{\beta}\right], V_{\gamma}\right\rangle
$$

where $A_{\mu}^{\alpha}$ is not a constrained field. By contrast with the integral (3.1), the action of the vector field within the inner product can generate only other vector fields on the sphere through the Lie derivative. However, the commutators also would give a combination of vector fields with coefficients related to the four-index symbol $\phi_{\alpha \beta \gamma \delta}$. The closure of the variation of the action under transformations of the gauge potential again require an infinite series of interaction terms.

The projection of these vector field, nevertheless, yields the $S U(2) \times S U(2)$ algebra together with a seventh nonlinear vector field [7]. There also exists a $G_{2}$-invariant Yang-Mills instanton in seven dimensions representing the internal coordinates of a heterotic string soliton [24], such that the $S U(2) \times S U(2)$ group after dimensional reduction over $S^{3}$ would be a nonperturbative symmetry in a field theory with potentials in the tangent bundle of $S^{7}$.

\section{Conclusion}

The equivalence of the residual symmetry group resulting from reduction of the transformation rule of a connection form in a bundle with a fibre diffeomorphic to a coset space and the isometry group as the structure group and the generalized dimensional reduction procedure for field theories is proven. The fibre-coordinate independence of the reduced transformation rule follows from the commutation of action of the parallelism with a subset of generators of the isometry group. Writing an element of the parallelism in terms of the coset $r S$, the relation $r s \cdot s^{\prime}=s^{\prime} r s^{\prime \prime}$ requires only $s^{\prime-1} r S s^{\prime}=r S$, which is satisfied by the centralizer of $C(R)$. Both methods yield a gauge group that is given by the centralizer of the tangent space group within the isometry group.

The exceptional bundle $E\left(M, \operatorname{Diff}\left(S^{1}\right) / S^{1}\right)$ does not belong to this category, because the fibre is $S^{1}$ rather than $\operatorname{Diff}\left(S^{1}\right) / S^{1}$. The existence of an infinitely generated residual symmetry group is equivalent to the action of the rotations along the B-cycles represented by generators of the Schottky group acting on a Riemann surface of infinite genus. The diffeomorphism on the boundary of the covering disk may be extrapolated to the interior, and therefore, the entire Riemann surface. The transformations on the surface which do not affect the Hilbert space of states on the ideal boundary then would be generated by this subgroup.

Supersymmetric theories admit a consistent dimensional reduction when the fibre is diffeomorphic to a parallelizable space. The residual symmetry group for the supersymmetric generalization of the exceptional bundle would be the supergroup $U(1 \mid 1) \times U(1 \mid 1) \times \ldots$. The projection to a subgroup generating $\operatorname{PSL}(2, R)$ would yield only three $U(1 \mid 1)$ factors. The effect of this supergroup on superstring states on the ideal boundary then may be determined.

Lagrangian field theories generally have Lie group symmetries and would not describe directly the phenomenology of the strong interactions related to an $S^{7}$ symmetry algebra. An integral with an invariant categorical form under transformations generated by global vector fields on the sphere may be found in the fibre bundle formulation. The nonassociativity of the octonion algebra, however, causes the occurrence of four-index symbols in the coefficients and nonvanishing of the variation of the action. An invariant action is constructed by introducing an infinite sequence of interaction terms in the integral and the transformation rules. The connection between this theory and elementary particle phenomenology will require the corresponding $(S U(3) \times U(1)) /\left(U(1)^{\prime} \times U(1)^{\prime \prime}\right)$ model for the fermions in the strong interactions.

\section{References}

1. Dreschler W, Mayer ME (1977) Fiber Bundles Techniques in Gauge Theories Lect Notes Phys, Springer, Berlin 67.

2. Manton N (1987) Connections on Discrete Fibre Bundles. Commun Math Phys 113: $341-351$.

3. Mangiarotti L, Sardanashvily G (2000) Connections in Classical and Quantum Field Theory, World Scientific, Singapore.

4. Bleecker D (1981) Gauge Theory and Variational Principles. Bull Amer Math Soc (N.S.) 9: 83-92.

5. Gelfand IM, Smirnov MM (1994) The Algebra of Chern-Simons Classes, the Poisson Bracket on it, and the Action of the Gauge Group. Lie Theory and Geometry, Progress in Mathematics, Birkhäuser, Boston 123: 261-288. 
Citation: Davis S (2016) Reduction over Coset Spaces and Residual Gauge Symmetry. J Generalized Lie Theory Appl 10: 249. doi:10.4172/17364337.1000249

Page 8 of 8

6. Davis S (1987) A Constraint on the Geometry of Yang-Mills Theory. J Geom Phys 4: 405-415.

7. Davis S (2005) Connections and Generalized Gauge Transformations. Int J Geom Meth Mod Phys 2: 505-542.

8. Davis S (1988) Coset Space of the Unified Field Theory. Class Quant Grav 5: 27.

9. Davis S (2011) String Compactifications and the Regge Trajectories for Resonances of the Strong Interactions. Int J Pure Appl Math 70: 25-38.

10. Manton N (1986) Dimensional Reduction of Supergravity. Ann Phys 167: 328-353.

11. Berkovits N (1990) A Geometrical Intepretation for the Symmetries of the Free Green-Schwarz Heterotic Superstring. Phys Lett B 241: 497-502.

12. Englert F, Sevrin A, Spindel Ph, Troost W, van Proeyen A (1988) Loop Algebras and Superalgebras based on S7. J Math Phys 29: 281-286.

13. Osipov EP (1988) Sugawara's Construction for Kac-Moody-Malcev Algebras. Phys Lett B 214: 371-373.

14. Adrianov AA, Novzhilov YV (1986) Correspondence Principle and Quantum Gauge theories with Chiral Fermions. Lett Math Phys 31: 199-207.

15. Wolf JA (1972) On the Geometry and Classification of Absolute Parallelisms. J Diff Geom 6: 317-342.
16. Helgason S (1984) Groups and Geometric Analysis, Academic Press, New York.

17. Kobayashi S (1972) Transformation Groups in Differential Geometry. SpringerVerlag, Berlin.

18. Uchida F (1975) Smooth Actions of Special Unitary Groups on Cohomology Complex Projective Spaces. Osaka J Math 12: 375-400.

19. Davis S, The Generalized Geometric Structure of String Theory.

20. Manton N (1981) Fermions and Parity Violation in Dimensional Reduction Schemes. Nucl Phys B 193: 502-516.

21. Geroch R (1968) Spinor Structure of Space-Time in General Relativity. J Math Phys 9: 1739-1744.

22. Petro J (1987) Great Sphere Fibrations of Manifolds. Rocky Mountain J Math 17: 865-886

23. Morita K (1981) Octonions, Quarks and QCD. Prog Theor Phys 65: 787-790.

24. Günaydin M, Nicolai H (1995) Seven-Dimensional Yang-Mills Instanton and its Extension to an Heterotic String Soliton. Phys Lett B 351: 169-172. 